\title{
Une nouvelle espèce cavernicole de Suisse Neobisium (N.) helveticum (Arachnide, Pseudoscorpion, Neobisiidae)
}

\author{
par

\section{J. HEURTAULT} \\ Muséum national d'histoire naturelle, Laboratoire de zoologie (Arthropodes) \\ 61 , rue Buffon. Paris $5^{\mathrm{e}}$. France.
}

Avec 1 planche

L'espèce est décrite d'après un exemplaire $q$ récolté par le $\mathrm{D}^{\mathrm{r}} \mathrm{V}$. Aellen en 1964 dans la grotte du Poteux (Valais).

\section{DESCRIPTION DE LA + HOLOTYPE}

Céphalothorax (fig. 1) à peine plus long que large. Deux paires d'yeux peu convexes, les yeux antérieurs distants de l'avant du céphalothorax d'une longueur sensiblement égale à leur diamètre. Trente et une soies céphalothoraciques (4 antérieures, 6 oculaires - auxquelles s'ajoutent des petites soies latérales entre les yeux et en avant de la première paire d'yeux -7 médianes, 14 (!) postérieures. Epistome bien développé en forme de triangle isocèle dont la base est 2 fois plus grande que la hauteur.

Soies tergales: 15.14 .13 .14 .14 .14 .17 .16 .16 .13$. Les soies inégales ne sont pas alignées régulièrement, elles augmentent de taille de l'avant vers l'arrière.

Sternites. Huit soies sur l'opercule génital disposées en une ligne courbe transverse. Plaque criblée médiane en forme de champignon. Vingt-trois soies alignées au bord postérieur du sternite 3 et 4 soies au-dessus de chaque stigmate 


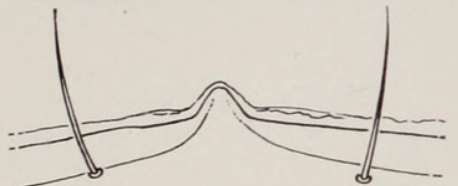

1 bis $\quad 0,1 \mathrm{~mm}$

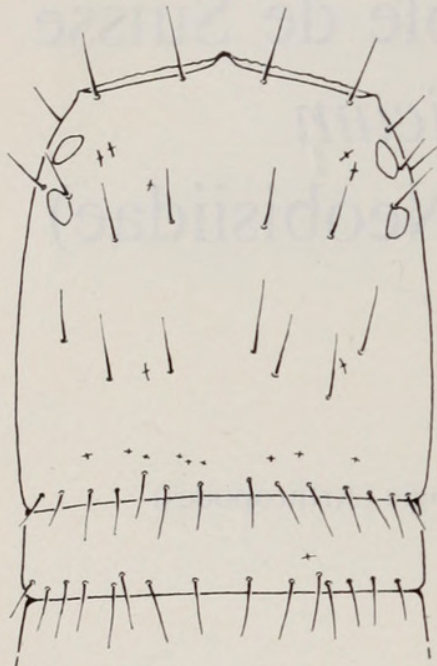

$1 \longmapsto 1 \mathrm{~mm}$
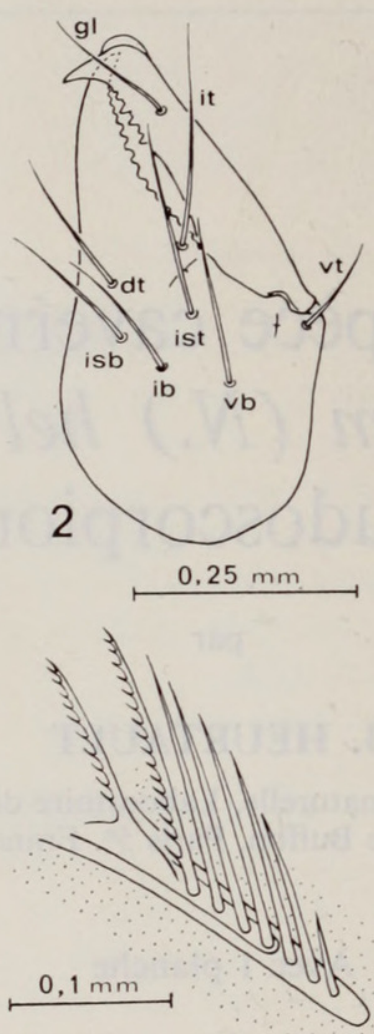

3
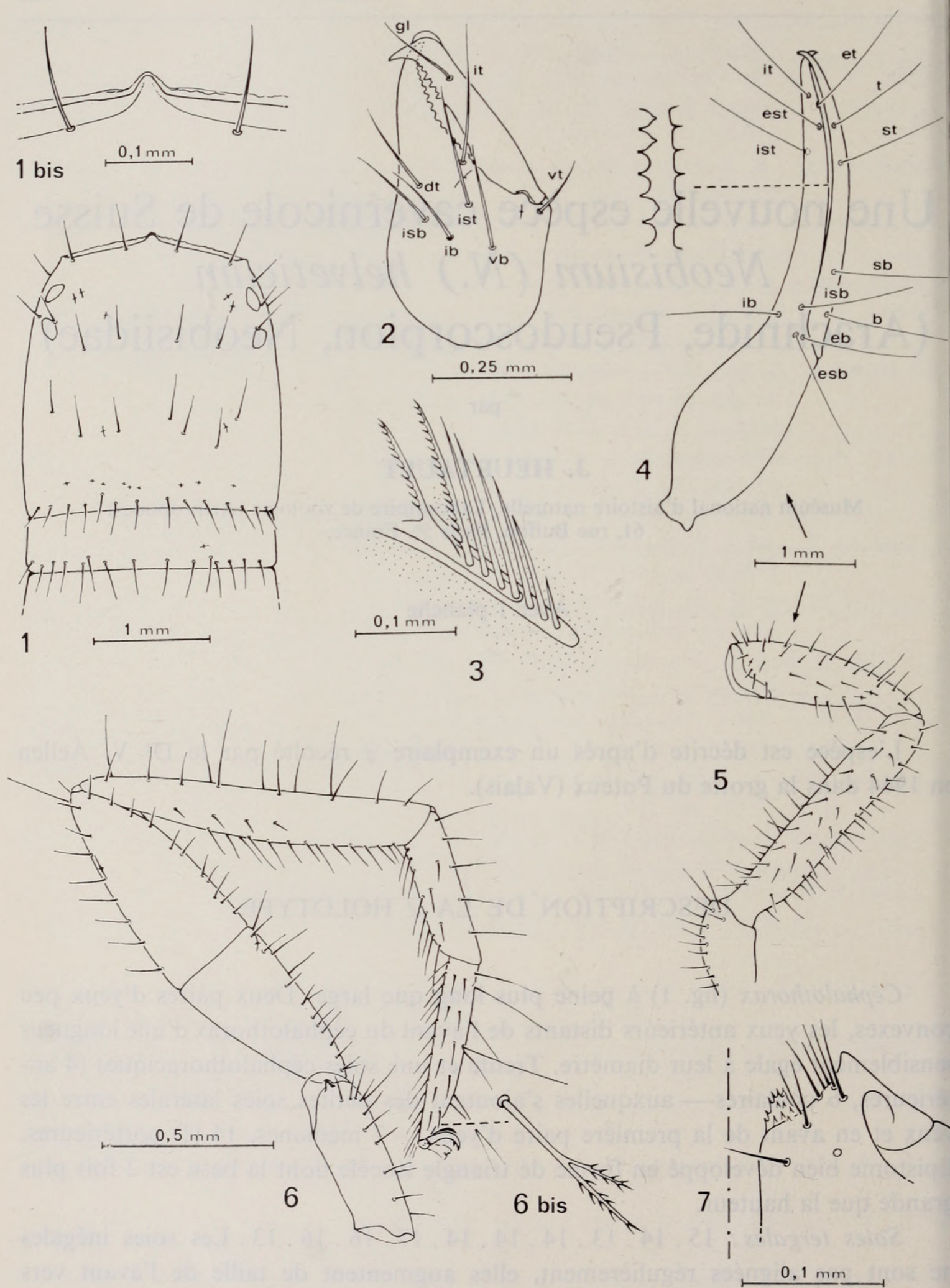

FIG. 1 à 9

Neobisium (N.) helveticum n. sp.

1: céphalothorax. 1 bis: épistome. 2 : chélicère droite, vue externe, $g l$ : soie galéale, $v t, v b:$ soies ventrale terminale, ventrale basale; it, ist, isb, $i b$ : soies intermédiaire terminale, intermédiaire sub-terminale, intermédiaire sub-basale, intermédiaire basale. 3: flagelle chélicérien. 4: pince droite, $t, s t, s b, b$ : soies terminale, sub-terminale, sub-basale, basale; $e t$, est, esb, $e b$ : soies externes terminale, sub-terminale, sub-basale, basale; $i t, i s t, i s b, i b$ : soies internes terminale, sub-terminale, sub-basale, basale. 5 : fémur et tibia de la pince droite. 6 : patte ambulatoire 4 . 6 bis: soie subterminale. 7: partie antérieure de la hanche 1. 
antérieur. Seize soies sur le sternite 4,3 et 2 soies au-dessus des stigmates postérieurs. Vingt-cinq soies sur le sternite 5. Dix-neuf soies sur les sternites 6, 7. Vingt et une soies sur le sternite 8 . Vingt soies sur le sternite 9. Seize soies sur le sternite 10.

Processus maxillaires avec 5 longues soies chacun.

Hanches des pattes-mâchoires : 17 et 18 soies. Seize et quinze soies aux hanches des pattes 1 dont l'angle distal interne se prolonge par un mamelon de chitine souple orné de spicules (fig. 7). Seize et quinze soies aux hanches des pattes 2. Onze et dix soies aux hanches des pattes 3 . Vingt soies aux hanches des pattes 4 .

Chélicères (fig. 2). Doigt mobile à tubercule fileur arrondi, pourvu de 8 canaux séricigènes terminaux. Cinq dents au doigt mobile séparées par des tubercules arrondis et 2 tubercules basaux. Six dents distales jointives au doigt fixe et 9 dents proximales écartées les unes des autres. Sept soies sur la main: vt, $v b$, it, ist, isb, ib (isb nettement écartée des 3 autres soies internes); $d t$. Flagelle typique des Neobisium: 8 soies inégales, les 2 distales dentelées, les autres simples, les 3 soies proximales isolées par leur insertion sur de la chitine dure, la soie distale est insérée sur un mamelon de chitine souple.

Pattes-mâchoires (fig. 4, 5). Trochanter avec 2 protubérances peu accentuées dont l'une porte une des deux lyrifissures. Fémur 5,8 fois plus long que large. Tibia 3,5 fois plus long que large, avec un pédoncule court, peu différencié et 2 lyrifissures au niveau de l'attache du pédoncule sur le tibia. Main 2,2 fois plus longue que large. Doigts égaux, 1,3 fois plus longs que la main avec pédoncule. Pince sans pédoncule, 4,8 fois plus longue que large. Tous les articles de la pattemâchoire sont lisses.

Trichobothries. $t$ au même niveau que est; $e b$ et esb très proches l'une de l'autre (fig. 4).

Denture. 81 dents au doigt mobile s'étendant jusqu'à la base du doigt, les 10 dents distales sont coniques et égales, les autres sont plates. Environ 90 dents au doigt fixe, inégales mais toujours coniques avec alternance irrégulière de grandes et de petites dents.

Pattes ambulatoires 1. 4 lyrifissures droites, presque parallèles sur la face interne du trochanter; préfémur avec une grande lyrifissure distale et une plus petite basale; télofémur avec 3 petites lyrifissures sur le tiers distal. Soies subterminales du tarse en $\mathrm{Y}$ à branches inégales et dentées.

Pattes ambulatoires 4 . 4 ou 5 lyrifissures parallèles groupées sur le quart distal interne postérieur du trochanter; une lyrifissure courbe près de la suture préfémur-télofémur, sur la face postérieure; 2 lyrifissures au tiers distal du télofémur sur la face antérieure. Fémur 4,3 fois plus long que large. Poil tactile inséré au milieu du tarse. Poil subterminal en $\mathrm{Y}$ à branches inégales dentées.

Dimensions en millimètres. Corps: 2,900; céphalothorax: 0,925; pattesmâchoires, fémur: $1,400-0,237$; tibia: $0,975-0,275$; pince sans pédoncule: 
2,350-0,487; main avec pédoncule: 1,075-0,487; doigt: 1,525 . Patte ambulatoire 1 préfémur: 0,675 ; télofémur: 0,450 ; tibia: 0,500 ; prétarse: 0,350 ; télotarse: $0,37 \xi$ Patte ambulatoire 4, fémur: $1,200-0,275$; tibia: $1,025-0,150$; prétarse: 0,475 télotarse: 0,575 .

\section{SITUATION DE NEOBISIUM (N.) HELVETICUM DANS} LA FAUNE EUROPÉENNE.

L'utilisation de la clef donnée en 1963 par M. BEIER (Ordnung Pseudo scorpionidea - Bestimmungsbücher Bodenfauna Europas. Berlin) permet d retenir les caractères suivants (p. 82 et suivantes):

$3^{*}$ ist du doigt fixe des pattes-mâchoires plus près de it que de ib et la plupart du temps distale par rapport au milieu du doigt. Pattesmâchoires effilées . . . . . . . . . . . . . . . . . 16

16* Fémur des pattes-mâchoires lisse, tout au plus pourvu de quelques petits tubercules grossiers épars . . . . . . . . . . . . . . . . . 24

24* Doigt mobile des pattes-mâchoires denté nettement jusqu'à la base . . 31

31 ist éloignée de $i b$ d'une distance inférieure au double de la distance ist - pointe du doigt . . . . . . . . . . . . . . . . . . . . . 32

32* Dents inégales au doigt fixe des pattes-mâchoires. Dents longues et dents courtes alternent dans la moitié distale du doigt . . . . . . . . 47

47 Epistome bien développé, saillant, plus ou moins pointu . . . . . . 48

48 . . . . . . . N. (N.) troglodytes (Beier, 1928)

$N$. (N.) jugorum (L. Koch, 1873)

N. (N.) noricum (Beier, 1939)

N. (N.) carpathicum (Beier, 1934)

La nouvelle espèce se différencie facilement des 4 espèces précédentes par uı fémur et un tibia plus élancés $(\mathrm{L} / 1$ du fémur $=5,8 ; \mathrm{L} / 1 \mathrm{du}$ tibia $=3,5)$, par u nombre de dents élevé au doigt fixe (supérieur à 90) des pattes-mâchoires. D’autre caractères vraisemblablement utilisables ne sont malheureusement pas connu chez les 4 espèces citées. Il s'agit de

- la formule chétotaxique céphalothoracique et tergale.

- la forme précise de l'épistome.

- la forme de l'appareil génital. 


\section{BIBLIOGR APHIE}

3EIER, M., 1963. Ordnung Pseudoscorpionidea. Bestimmungsbücher Bodenfauna Europas. Berlin.

IACHON, M. et P. D. GabButT., 1964. Sur l'utilisation des soies flagellaires chélicériennes dans la distinction des genres Neobisium J. C. Chamberlin et Roncus L. Koch. (Arachnides, Pseudoscorpions, Neobisiidae). Bull. Soc. Zool. Fr., 89: 174-188. 


\section{$2 \mathrm{BHL}$ Biodiversity Heritage Library}

Heurtault, J. 1971. "Une nouvelle espèce cavernicole de Suisse Neobisium (N.) helveticum (Arachnide, Pseudoscorpion, Neobisiidae)." Revue suisse de zoologie 78, 903-907. https://doi.org/10.5962/bhl.part.97082.

View This Item Online: https://www.biodiversitylibrary.org/item/138401

DOI: https://doi.org/10.5962/bhl.part.97082

Permalink: https://www.biodiversitylibrary.org/partpdf/97082

\section{Holding Institution}

American Museum of Natural History Library

\section{Sponsored by}

BHL-SIL-FEDLINK

\section{Copyright \& Reuse}

Copyright Status: Public domain. The BHL considers that this work is no longer under copyright protection.

Rights Holder: Muséum d'histoire naturelle - Ville de Genève

This document was created from content at the Biodiversity Heritage Library, the world's largest open access digital library for biodiversity literature and archives. Visit BHL at https://www.biodiversitylibrary.org. 